\title{
The Dynamics of Cortical and Hippocampal Atrophy in Alzheimer Disease
}

Mert R. Sabuncu, PhD; Rahul S. Desikan, MD, PhD; Jorge Sepulcre, MD, PhD; Boon Thye T. Yeo, PhD; Hesheng Liu, PhD; Nicholas J. Schmansky, MSc; Martin Reuter, PhD; Michael W. Weiner, MD; Randy L. Buckner, PhD; Reisa A. Sperling, MD; Bruce Fischl, PhD; for the Alzheimer's Disease Neuroimaging Initiative

Objective: To characterize rates of regional Alzheimer disease (AD)-specific brain atrophy across the presymptomatic, mild cognitive impairment, and dementia stages.

Design: Multicenter case-control study of neuroimaging, cerebrospinal fluid, and cognitive test score data from the Alzheimer's Disease Neuroimaging Initiative.

Setting: Research centers across the United States and Canada.

Patients: We examined a total of 317 participants with baseline cerebrospinal fluid biomarker measurements and $3 \mathrm{Tl}$ weighted magnetic resonance images obtained within 1 year.

Main Oufcome Measures: We used automated tools to compute annual longitudinal atrophy in the hippocampus and cortical regions targeted in AD. We used MiniMental State Examination scores as a measure of cognitive performance. We performed a cross-subject analysis of atrophy rates and acceleration on individuals with an AD-like cerebrospinal fluid molecular profile.

Results: In presymptomatic individuals harboring indicators of $\mathrm{AD}$, baseline thickness in $\mathrm{AD}$-vulnerable cor- tical regions was significantly reduced compared with that of healthy control individuals, but baseline hippocampal volume was not. Across the clinical spectrum, rates of AD-specific cortical thinning increased with decreasing cognitive performance before peaking at approximately the Mini-Mental State Examination score of 21, beyond which rates of thinning started to decline. Annual rates of hippocampal volume loss showed a continuously increasing pattern with decreasing cognitive performance as low as the Mini-Mental State Examination score of 15. Analysis of the second derivative of imaging measurements revealed that AD-specific cortical thinning exhibited early acceleration followed by deceleration. Conversely, hippocampal volume loss exhibited positive acceleration across all study participants.

Conclusions: Alzheimer disease-specific cortical thinning and hippocampal volume loss are consistent with a sigmoidal pattern, with an acceleration phase during the early stages of the disease. Clinical trials should carefully consider the nonlinear behavior of these AD biomarkers.

Arch Neurol. 2011;68(8):1040-1048
Author Affiliations are listed at the end of this article. Group Information: A list of the Alzheimer's Disease Neuroimaging Initiative members appears at http://adni.loni.ucla.edu /about/who-we-are /principal-investigators.

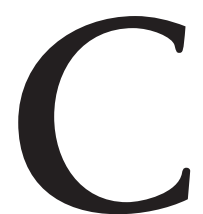

OGNITIVE DECLINE AND the dementia stage of Alzheimer disease (AD) are the clinical manifestations of the cumulative burden of multiple neuropathologic insults. Postmortem studies ${ }^{1,2}$ indicate that an evolution of neuropathologic insults can be observed during the initial stages of $\mathrm{AD}$, with intracellular tau-associated neurofibrillary tangles first appearing in medial temporal and limbic isocortical regions and extracellular amyloid- $\beta$ (A $\beta)$-associated plaques affecting heteromodal association cortices.

Structural magnetic resonance imaging (MRI) allows for the detection of macroscopic tissue atrophy associated with AD. Serial imaging helps us view the temporal evolution of the disease process and shows promise for use in assessing drug efficacy. ${ }^{3-14}$ Most prior longitudinal studies have found that brain atrophy progres- sively accelerates throughout the disease process, although those studies mostly focus on global measures, such as wholebrain or ventricular volume, ${ }^{3}$ or on a single anatomical structure, such as the hippocampus. ${ }^{5,10,12}$

In this study, we used longitudinal MRI to examine the temporal dynamics of regional cortical and hippocampal atrophy in individuals harboring indicators of AD. Gray matter atrophy, resulting from the loss of neuronal, glial, and neuropil volume, is reflected as diminished cortical thickness in vulnerable regions ${ }^{15,16}$ and decreased volume of the hippocampus. ${ }^{17}$ Building on the findings of prior longitudinal clinical, cognitive, and imaging studies ${ }^{3,7,18-22}$ and a recent theoretical model of the Alzheimer's pathologic cascade, ${ }^{23}$ we hypothesized that AD-specific cortical and hippocampal atrophy follows a sigmoidal pattern with initial acceleration followed by deceleration during the later stages of the disease. Our 
analyses revealed that hippocampal and AD-specific cortical atrophy agree well with the cumulative diffusion model that predicts a sigmoidal pattern. We further present 3 lines of evidence (based on baseline measurements, atrophy rates, and acceleration) that distributed AD-specific cortical thinning constitutes an early biomarker of AD.

\section{METHODS}

\section{CUMULATIVE DIFFUSION MODEL}

Recent evidence suggests that the dynamics of AD biomarkers follow a sigmoidal pattern. ${ }^{23,24}$ One mechanism that yields such a dynamic is the cumulative diffusion model,${ }^{25}$ which predicts that the rate of atrophy is proportional to that of aggregated atrophy (ie, tissue loss at a location is aggravated by accumulating damage in its neighborhood). Underlying this model is the cumulative damage hypothesis, which can be caused by mechanisms such as oxidative stress. ${ }^{26}$

According to the cumulative diffusion model, atrophy accelerates initially, and the rate peaks at a point at which half the potential tissue loss has occurred (Figure 1). This critical (ie, inflection) point marks a shift in dynamics, namely, before the inflection atrophy is driven by accumulating disease, but in the following period, the rate is constrained by the amount of intact tissue.

\section{ALZHEIMER'S DISEASE NEUROIMAGING INITIATIVE DATA}

We examined 317 participants from the public Alzheimer's Disease Neuroimaging Initiative (ADNI) database (http://www .adni-info.org), with cerebrospinal fluid (CSF) samples obtained at baseline and 3 T1-weighted MRIs acquired at baseline and at 6 and 12 months of follow-up. We investigated CSF biomarker measurements of A $\mathrm{A} 1-42$ peptide and total tau (t-tau) according to a recently established CSF signature of AD ${ }^{27}$ These measurements, which we consider to be indirect measures of indicators of $\mathrm{AD}$, show strong promise as preclinical biomarkers that predict future dementia in individuals without dementia. ${ }^{26-28}$ Our analysis focused on the group of individuals with a CSF molecular profile consistent with AD (ie, a CSF t-tau: $A \beta 1-42$ ratio $>0.39)(N=202)$. This group contained presymptomatic (ie, cognitively normal $[\mathrm{CN}]$ as measured by a Clinical Dementia Rating ${ }^{29}$ of 0$)$ individuals $(n=31)$, patients with amnestic mild cognitive impairment ${ }^{30}(\mathrm{aMCI})(\mathrm{n}=107)$, and patients with $\operatorname{AD}(n=64)$. Also, as healthy control individuals $(n=61)$, we included those individuals who were $\mathrm{CN}$ at baseline and had a normal CSF molecular profile (ie, a CSF t-tau: A $\beta 1-42$ ratio < 0.39). The Table lists descriptive information for these groups.

\section{IMAGE PROCESSING}

We processed all MRIs automatically using the FreeSurfer software package developed at the A. A. Martinos Center for Biomedical Imaging at Massachusetts General Hospital. ${ }^{1}$ For each MRI result, FreeSurfer computed study participant-specific thickness measurements across the entire cortical mantle $e^{31,32}$ and hippocampal and intracranial volume measurements. ${ }^{33,34}$ In all subsequent analyses, we averaged the volumes of the 2 hippocampi. We used FreeSurfer's longitudinal stream to process a set of serial MRIs from each study participant; this stream yields accurate and unbiased estimates of subtle changes over time. $^{2}$
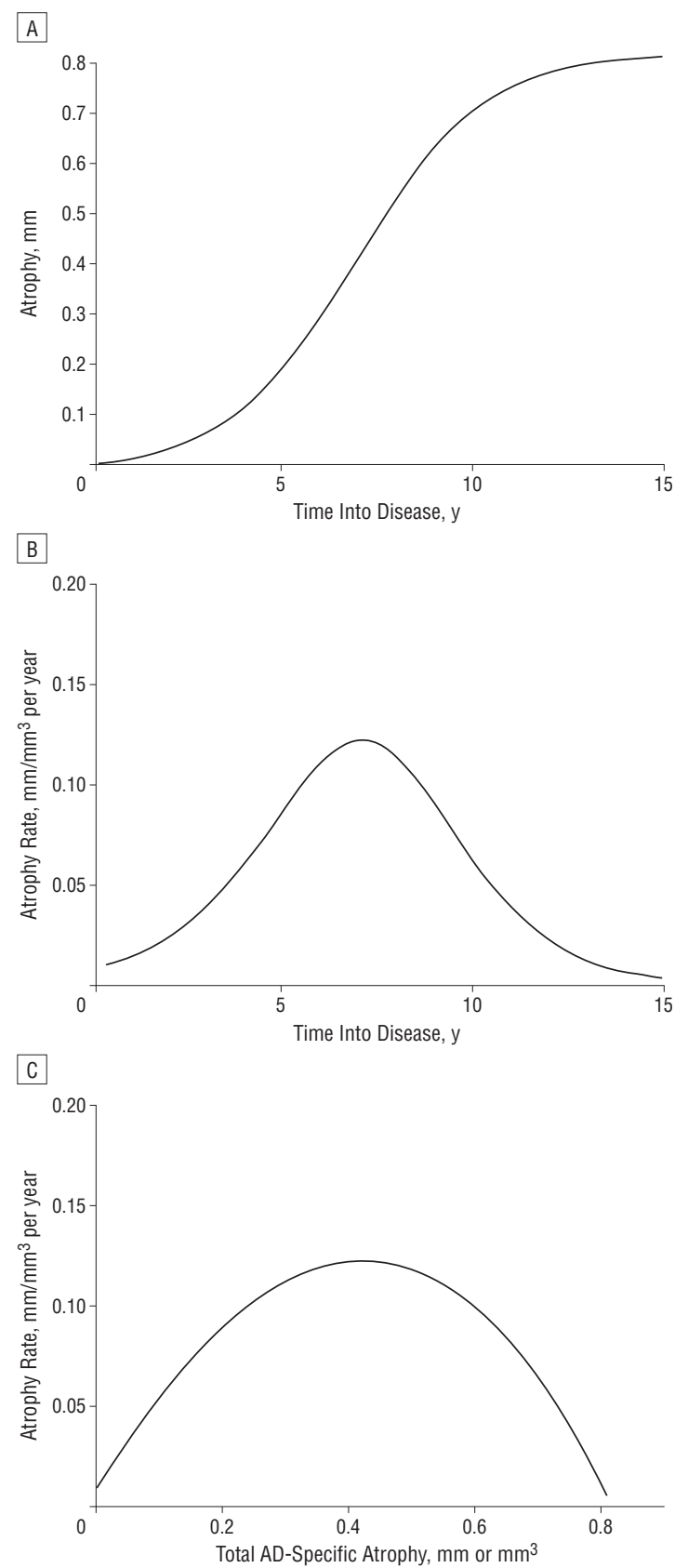

Figure 1. Rates of atrophy. A, Hypothetical curve of longitudinal atrophy according to the cumulative diffusion model; $B$, corresponding rate of atrophy; and $C$, rate of atrophy as a function of the total amount of Alzheimer disease $(A D)$-specific atrophy.

\section{CORTICAL REGIONS OF INTEREST}

We used the cross-sectional Open Access Series of Imaging Studies (OASIS) data set $\mathrm{t}^{35}$ consisting of 94 participants to generate an exploratory map of cortical thickness differences between older controls (47; [57.4\%] women; mean [SD] age, 78 [5.6] years) and individuals with a Clinical Dementia Rating of 0.5 clinically classified as having incipient AD (47; [63.8\%] women; 76.4 [4.7] years of age). A 2-class general linear model yielded a map of regions exhibiting $\mathrm{AD}$-specific thinning $(P<.01$, uncorrected) in each hemisphere (Figure 2). We delineated 7 regions of interest (ROIs) on the average cortical surface tem- 
Table. Descriptive Baseline Statistical Information for the Study Participants

\begin{tabular}{|c|c|c|c|c|c|}
\hline \multirow[b]{2}{*}{ Variable } & \multirow{2}{*}{$\begin{array}{c}\text { Control } \\
\text { Individuals } \\
(\mathrm{n}=61)\end{array}$} & \multicolumn{4}{|c|}{ AD-Like CSF } \\
\hline & & $\begin{array}{l}\text { Presymptomatic } \\
\qquad(n=31)\end{array}$ & $\begin{array}{c}\text { aMCI } \\
(n=107)\end{array}$ & $\underset{(n=64)}{A D}$ & $\begin{array}{l}\text { Total AD-Like CSF } \\
\qquad(\mathrm{n}=202)\end{array}$ \\
\hline Age, mean (SD), y & $67.2(12.0)$ & $68.5(9.7)$ & $66.9(13.2)$ & $68.1(12.8)$ & $67.5(12.6)$ \\
\hline Female sex, №. (\%) & $46(75.4)$ & $23(74.2)$ & 78 (72.9) & 39 (60.9) & $140(69.3)$ \\
\hline MMSE score, mean (SD) & $29.1(1.0)$ & $29.2(1.1)$ & $26.9(1.8)$ & $23.3(1.9)$ & $26.1(2.7)$ \\
\hline Educational level, mean (SD), y & $15.3(2.5)$ & $16.3(2.5)$ & $15.6(2.5)$ & $15.1(2.8)$ & $15.5(2.6)$ \\
\hline Hypertension, №. (\%) & $31(50.8)$ & $15(48.4)$ & $42(39.3)$ & 30 (46.9) & $87(43.1)$ \\
\hline 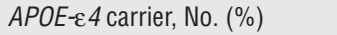 & $9(14.8)$ & $11(35.5)$ & $70(65.4)$ & $46(71.9)$ & $127(62.9)$ \\
\hline
\end{tabular}

Abbreviations: AD, Alzheimer disease; aMCI, amnestic mild cognitive impairment; APOE, apolipoprotein E; CSF, cerebrospinal fluid; MMSE, Mini-Mental State Examination.
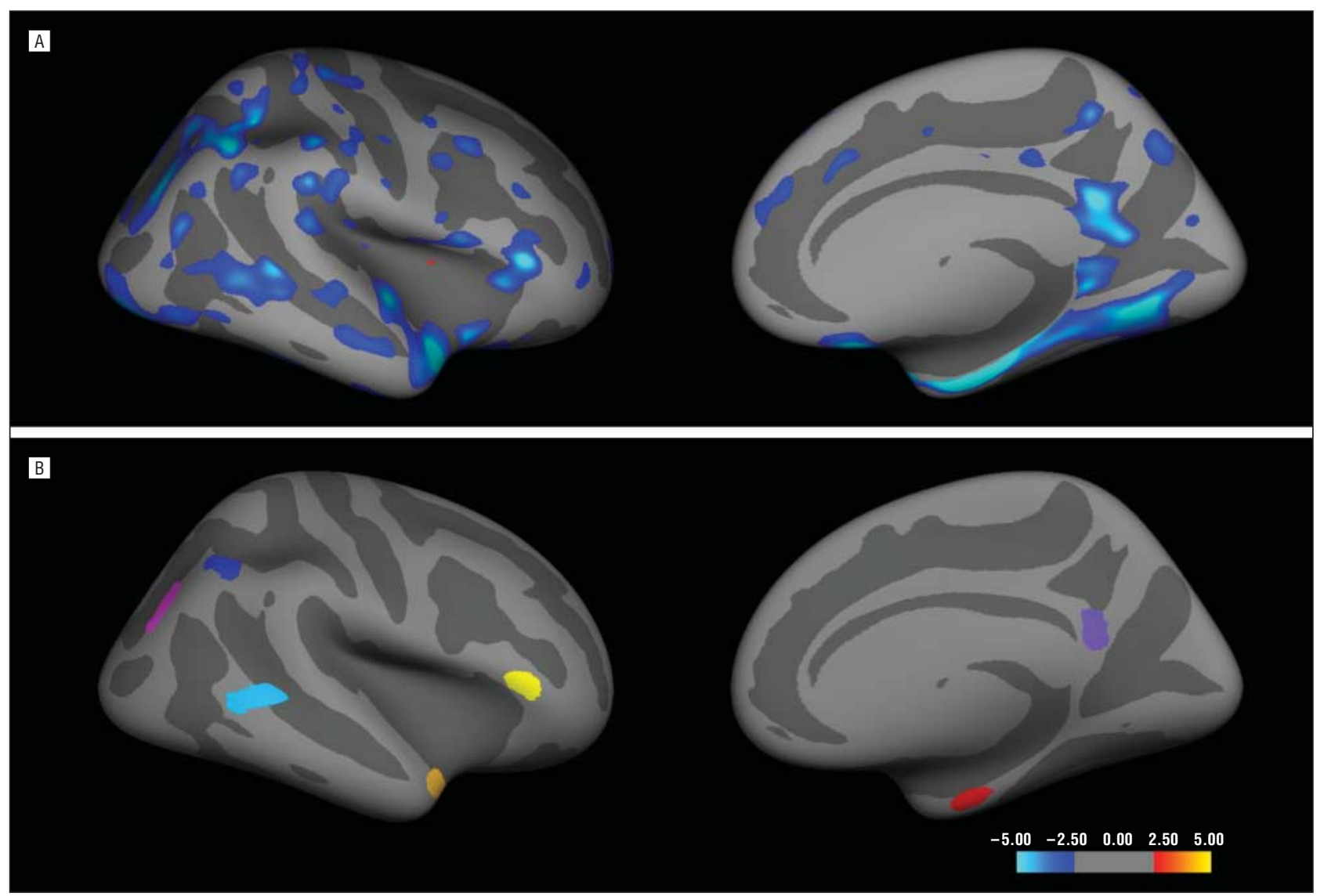

Figure 2. Seven regions of interest generated from the regions demonstrating the greatest magnitude of Alzheimer disease (AD)-specific thinning in the exploratory analysis of the Open Access Series of Imaging Studies data, shown on an inflated surface representation, with dark gray regions representing sulci and light gray regions representing gyri. For illustration purposes, only the right hemisphere is presented. A, Statistical significance values of the difference between healthy control individuals and patients with incipient AD. This exploratory analysis was not corrected for multiple comparisons; therefore, this map is not intended to be an accurate reflection of early AD-specific thinning. However, the overall pattern is in general agreement with reported results. $B$, The regions of interest.

plate $^{31}$ that demonstrated the greatest magnitude of bilateral cortical thinning in participants with incipient AD relative to older controls (Figure 2). These regions were the entorhinal cortex, the temporopolar cortex, the lateral temporal cortex, the inferior parietal cortex, the inferior parietal sulcus, the posterior cingulate cortex, and the inferior frontal cortex. Also, we used a primary motor and sensory cortex region to serve as a control ROI by averaging the thickness of the precentral and postcentral gyri. ${ }^{26}$

Using surface-based registration, ${ }^{36}$ we mapped the OASIS-derived ROIs from the surface template onto the Freesurfer-generated results of the individual participants from the
ADNI cohort. We used mean thickness values (ie, across the cortical ROIs in both hemispheres) in all subsequent analyses (unless specified otherwise).

\section{RATES OF ATROPHY}

We computed rates of AD-specific cortical thinning and hippocampal volume loss in each participant (at month 6) as the mean of the backward (ie, baseline-month 6) and forward (ie, month 6-month 12) slopes of cortical thickness and hippocampal volume measurements, respectively. With 2 time points, 
the slope equals the difference between measurements divided by the time difference. We computed the second derivative with respect to time (ie, acceleration) as the difference between the forward and backward slopes divided by the time difference between month 12 and baseline. These values were used in the acceleration-deceleration analysis presented herein. Positive acceleration indicates that atrophy is speeding up, and negative acceleration (ie, deceleration) signifies that it is slowing down.

\section{LONGITUDINAL MINI-MENTAL STATE EXAMINATION SCORES}

We measured cognitive performance via Mini-Mental State Examination (MMSE) scores ${ }^{37}$ obtained at baseline, month 6 , and month 12. Annual cognitive decline was computed as the difference between the scores of baseline and month 12 divided by the time difference.

\section{STATISTICAL ANALYSIS}

To evaluate the fit of the cumulative diffusion model on individual-level data, we used a model selection strategy based on stepwise logistic regression. ${ }^{38}$ The theoretical model predicts a nonlinear, quadratic relationship between the rate of atrophy and the total amount of AD-specific atrophy (Figure 1C). We used cognitive performance (ie, negative MMSE score) as a proxy for the total amount of $\mathrm{AD}$-specific atrophy. The rate of atrophy was treated as the dependent (ie, measurement) variable in a general linear model, with MMSE score (at month 6 of follow-up) and its square as independent variables of interest. We included age, sex, educational level, apolipoprotein E (APOE)- $\varepsilon 4$ genotype (using 1 if an APOE- $\varepsilon 4$ carrier and 0 otherwise), and intracranial volume as covariates.

For a given measurement (ie, cortical thickness or hippocampal volume), if the rate of atrophy is associated with the square of the MMSE score via a negative coefficient, we consider this to be evidence of the cumulative diffusion model (ie, outcome 1 ). We further consider the outcome in which the rate of atrophy has a linear (and not quadratic) association with MMSE score via a negative coefficient (ie, outcome 2 ) as supportive of the cumulative model because it simply would indicate that the inflection point is beyond the (MMSE) range of the analyzed data. Our assumption is that continuously increasing atrophy rates will slow at some point due to the diminishing amount of intact tissue. All other outcomes will be indicative of an alternative mechanism (ie, alternative outcome). For example, a progressive slowing will suggest an exponential decay pattern. All our analyses included age, sex, educational level, APOE- $\varepsilon 4$ genotype, and intracranial volume as covariates and were conducted using the Statistical Toolbox of Matlab (R2007a; The MathWorks Inc, Natick, Massachusetts).

\section{RESULTS}

\section{ATROPHY IN PRESYMPTOMATIC INDIVIDUALS HARBORING INDICATORS OF AD}

Among individuals with an AD-like CSF molecular profile, we first analyzed those who were $\mathrm{CN}$ at baseline $(n=31)$. Compared with healthy controls, the mean baseline thickness in the $7 \mathrm{AD}$-vulnerable cortical ROIs was significantly reduced in this presymptomatic group $(P<.001)$. Yet, baseline hippocampal volume measurements and the annual rates of AD-specific cortical thin- ning and hippocampal volume loss were statistically indistinguishable from those of healthy controls $(P=.17$, $P=.81$, and $P=.24$, respectively) (Figure 3). A correlation analysis that treated CSF biomarkers as continuous variables in the entire $C N$ group $(n=92)$ revealed that mean baseline thickness in AD-vulnerable cortical ROIs was more strongly associated with $\mathrm{A} \beta 1-42$ than $\mathrm{t}$-tau, and this association mostly was driven by a subset of the 7 regions, with posterior cingulate and inferior parietal cortices exhibiting the strongest correlations. ${ }^{26}$ The mean results agree well with supplemental analyses performed on a small number of $\mathrm{CN}$ individuals who progressed to aMCI or AD within 3 years. ${ }^{26}$ Furthermore, among the presymptomatic individuals, annual rates of ADspecific cortical thinning and hippocampal volume loss were not correlated with concurrent cognitive decline (ie, MMSE change $e^{3}$ during the same year; partial correlation $P=.57$ and $P=.63$, respectively).

\section{ATROPHY IN SYMPTOMATIC INDIVIDUALS HARBORING INDICATORS OF AD}

Next, we examined the symptomatic individuals (ie, those having aMCI and AD) with an AD-like CSF molecular profile. Compared with healthy controls, thickness in ADvulnerable cortical ROIs and hippocampal volume were significantly reduced in this group (both $P<.001$ ). The rates of $\mathrm{AD}$-specific thinning and hippocampal volume loss were significantly higher than those in the control group (both $P<.001$ ) and correlated with concurrent cognitive decline (ie, partial correlations of 0.16 and 0.32 ; corresponding $P=.02$ and $P<.001$ for cortical thickness and hippocampal volume, respectively).

\section{ANNUAL RATES OF ATROPHY VS MMSE SCORE}

To test the cumulative diffusion model of atrophy, we conducted a stepwise linear regression on all individuals with an AD-like CSF molecular profile $(n=202)$, using rate of atrophy as the outcome variable. The MMSE score at month 6 and its square were the independent variables of interest. The quadratic term was significantly associated with the rate of AD-specific cortical thinning $(P=.002)$ via a negative coefficient (ie, outcome 1$)$. No significant association was observed between the square of the MMSE score and the annual rate of hippocampal volume loss $(P=.76)$, but the linear term was statistically significant $(P<.001)$ with a negative coefficient (ie, outcome 2). Both of these outcomes provide support for the cumulative diffusion model that predicts a sigmoidal pattern. For the primary motor and sensory cortices (ie, the control region), stepwise linear regression yielded no support for the cumulative diffusion model (ie, the alternative outcome). ${ }^{26}$ Figure 4 shows the best secondorder polynomial fit for AD-specific cortical thinning rates and hippocampal volume loss rates as functions of MMSE score.

The maximum point of the thinning rate curve was located at the MMSE score of 21.4, with a 95\% confidence interval of 19.5 to 23.3 , a range that is typically within the mild stages of clinical AD. ${ }^{4}$ To exclude the possibility that this fit may have been driven by results from 


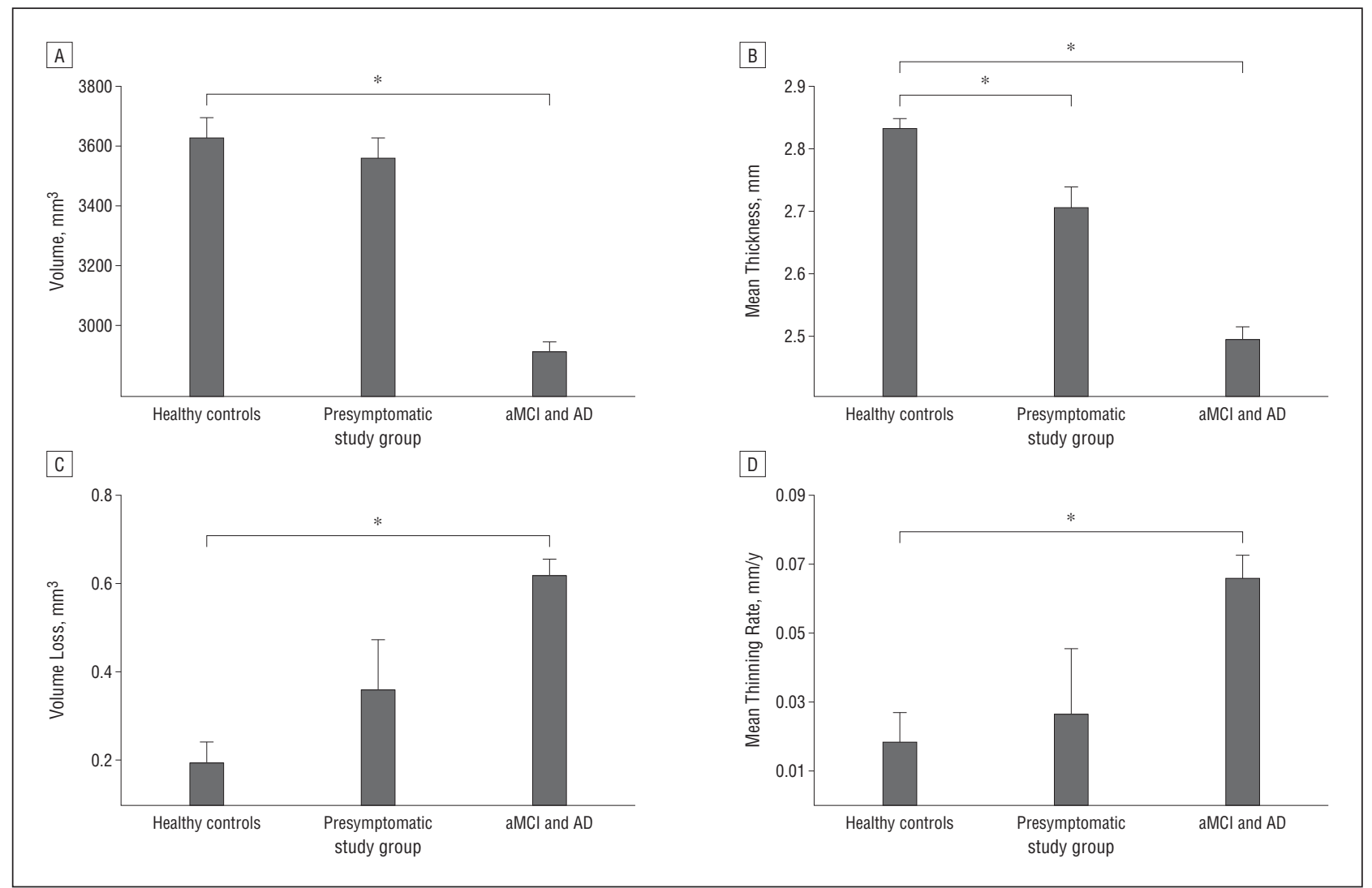

Figure 3. Baseline hippocampal volume measurements and the annual rates of Alzheimer disease (AD)-specific cortical thinning and hippocampal volume loss. Shown are mean hippocampal volume $(A)$ and cortical thickness in AD-vulnerable regions of interest (B) across healthy control individuals, presymptomatic individuals, and symptomatic (ie, having amnestic mild cognitive impairment $[\mathrm{aMCl}]$ and $\mathrm{AD}$ ) individuals with an $\mathrm{AD}$-like cerebrospinal fluid molecular profile. Mean rates of hippocampal volume loss (C) and AD-specific cortical thinning (D) are shown for each group. Error bars indicate SEM. Asterisk indicates $P<.05$ for group differences.

the entorhinal cortex, we conducted a supplemental analysis by omitting this region from the average AD-specific thinning measure. ${ }^{26}$ Little variation can be observed in the location of the peak across the $7 \mathrm{AD}$-vulnerable ROIs, with less than 2 MMSE points of difference between the maxima of the posterior cingulate, the ROI that peaks the earliest, and the temporal pole, which peaks the latest. ${ }^{26}$ The rate of hippocampal volume loss exhibited a continuously increasing pattern with decreasing MMSE score, and the maximum of this curve is likely to exist beyond the MMSE score of 15 (ie, the lowest score in our data).

\section{ACCELERATION AND DECELERATION OF ATROPHY}

The sigmoidal pattern suggests early acceleration followed by a deceleration phase. Based on the observation that AD-specific cortical thinning rates peak at approximately the MMSE score of 20 to 23, we subdivided the group of individuals with an AD-like CSF molecular profile into 2 subgroups, namely, those with a month-6 MMSE score greater than $23(n=148)$ and those whose score was less than $20(n=10)$. Cortical atrophy exhibited a pattern of acceleration in the first group (ie, the second derivative is positive) and deceleration in the second group. ${ }^{26}$ Conversely, hippocampal volume loss exhibited a positive acceleration in both subgroups.

\section{COMMENT}

We present evidence for the cumulative diffusion model as a possible mechanism for AD-specific brain atrophy. In this model, rates of atrophy behave nonlinearly (ie, with a sigmoidal pattern), increasing initially, peaking at the inflection point, and decreasing thereafter.

Rates of atrophy in AD-vulnerable cortical ROIs and in the hippocampus in presymptomatic individuals with an AD-like CSF molecular profile were statistically indistinguishable from those of healthy controls. However, the former individuals exhibited an elevated profile, suggesting that these 2 groups can be potentially discriminated with a larger sample size. Of interest, the mean thickness of AD-vulnerable ROIs was significantly reduced in this presymptomatic group, but hippocampal volume was not. This finding suggests that cortical thickness in AD-vulnerable ROIs may be a sensitive biomarker in the earliest stages of the disease process. Longitudinal rates of atrophy in AD-vulnerable cortical and hippocampal regions, however, seem unlikely to be useful for tracking disease progression during the presymptomatic period. These results are further supported by a supplemental analysis of a small number of individuals $(\mathrm{n}=10)$ who were $\mathrm{CN}$ at baseline but had progressed to aMCI or AD within 3 years of follow-up. 
Our results from the $\mathrm{CN}$ group add to the growing body of literature that demonstrates cortical thickness measurements from select ROIs as sensitive markers of very early $\mathrm{AD} .{ }^{39-41}$ This observation does not necessarily contradict the widely accepted pathophysiology of AD that is characterized by early neurofibrillary tangle deposition in the medial temporal lobe (including the hippocampus) and associated atrophy in these regions because volume measurements of a structure as large as the hippocampus may be less sensitive to subtle and localized atrophy than those of ROI-based cortical thickness. Moreover, our analyses suggest that AD-specific cortical thinning in $\mathrm{CN}$ individuals is mostly associated with $\beta$-amyloid and not tau. The recently demonstrated tight coupling between hippocampal atrophy and episodic memory impairment ${ }^{42}$ further suggests that significant volume loss in the hippocampus may be associated with clinical symptoms and therefore is unlikely to occur during a preclinical phase. That is, hippocampal atrophy may mark the transition to cognitive symptoms.

Thickness in AD-vulnerable cortical ROIs and hippocampal volume are significantly reduced during aMCI and clinical AD. Consistent with data from prior studies, ${ }^{5-8,18}$ rates of AD-specific cortical thinning and hippocampal volume loss also are significantly elevated and correlate with concurrent cognitive decline.

Consistent with the sigmoidal pattern, the rate of cortical thinning accelerates throughout the presymptomatic and aMCI stages, starting from levels indistinguishable from those of healthy controls and reaching its fastest pace at approximately the MMSE score of 21. Although continuing to progress, AD-specific cortical thinning starts to slow beyond this point. This characterization was consistent across all 7 ADvulnerable cortical ROIs that we examined. Hippocampal atrophy rates, however, exhibit a progressively increasing pattern without a clearly discernible peak before the MMSE score of 15.

Our longitudinal observation is in broad agreement with a recent cross-sectional characterization of the dynamics of AD biomarkers, ${ }^{24}$ in which a sigmoidal pattern of hippocampal atrophy was demonstrated. Building on data from prior serial imaging studies, ${ }^{7,14,18}$ our results demonstrate that $\mathrm{AD}$-specific brain atrophy is characterized by early acceleration, possibly driven by cumulative insults, such as amyloid toxicity, tangle deposition, and neuronal and synaptic dysfunction, followed by late deceleration, constrained by the diminishing residual intact tissue.

The nonlinear, sigmoidal pattern has important implications for clinical trials. First, a linear characterization of brain atrophy can lead to incorrect sample size estimates and underpowered clinical trials. Second, the bell-shaped derivative of the sigmoid implies that early in the disease process, atrophy rates are likely to be indistinguishable from those of controls and therefore probably will be of limited use in tracking progression. Finally, the natural deceleration observed in later disease stages needs to be carefully considered when assessing a disease-modifying therapeutic effect in an AD trial.

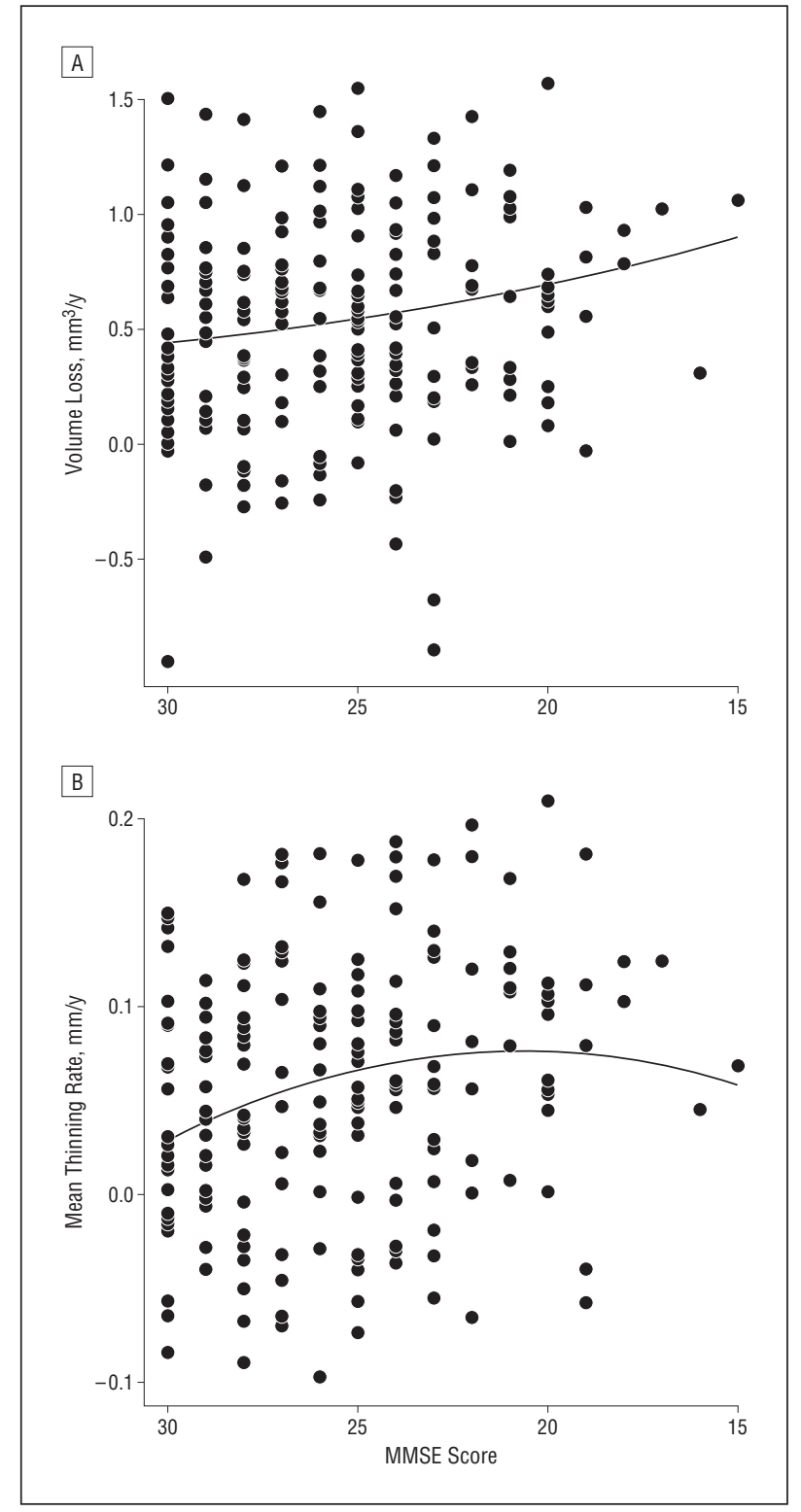

Figure 4. Best second-order polynomial fit for Alzheimer disease (AD)-specific cortical thinning rates and hippocampal volume loss rates as functions of Mini-Mental State Examination (MMSE) score. Shown are scatterplots of rates of AD-specific hippocampal volume loss (A) and cortical thinning (B) vs MMSE score. Each dot represents an individual with an AD-like cerebrospinal fluid molecular profile. The curves represent best-fit quadratic functions. The curve for the hippocampus is approximately linear (but slightly convex due to a nonsignificant positive coefficient in the quadratic term). The curve for AD-specific cortical thinning is concave, with an optimum at approximately the MMSE score of 21. To examine the sensitivity of the fit to outliers, we reanalyzed these data without the 2 individuals with extreme data, namely, those with MMSE scores of 16 and 15, respectively. All results remained approximately the same. Crucially, the quadratic term for AD-specific thinning was statistically significant, and the optimum score was approximately 21 .

The present study uses longitudinal neuroimaging data collected from multiple sites and thus demonstrates the potential use of these biomarkers in multicenter clinical trials. Another important aspect of the study is the use of cortical ROIs defined with an independent sample. In contrast with traditional methods that use anatomical landmarks, this approach identifies a disease-specific effect through an exploratory analysis ${ }^{39}$ which yields sensitive markers of disease. 
The present study has several limitations. One issue involves the use of a cross-sectional design in which cohort effects can confound results. A second concern pertains to the usefulness of a CSF-based cutoff to select participants who have a molecular profile consistent with $\mathrm{AD}$ and our assumption that the conditions of these individuals exist on the same disease trajectory. Hence, our findings are contingent on the validity of this hypothetical trajectory. Another limitation involves the examination of longitudinal atrophy during a 1-year period. It is possible that acceleration and deceleration patterns of cortical regions vary substantially across individuals. Furthermore, the limited number of patients in the severe stages of dementia (eg, with an MMSE score <20) may have biased our computation of where cortical thinning rates peak. As additional longitudinal MRI data during multiyear periods become available, future studies will examine the validity of these findings. Finally, the apparent slowing of AD-specific cortical thinning may not occur due to the underlying biology but may be a consequence of the technical difficulty of resolving thickness changes around and beyond the voxel resolution. Although our characterization of AD-specific cortical thinning as a dynamic biomarker still will be valid, in the interest of understanding the underlying biology of this region, this potential confounder needs to be examined in future work.

Accepted for Publication: October 28, 2010.

Author Affiliations: Athinoula A. Martinos Center for Biomedical Imaging, Departments of Radiology, Massachusetts General Hospital, Charlestown (Drs Sabuncu, Desikan, Sepulcre, Yeo, Liu, Reuter, Buckner, Sperling, and Fischl and Mr Schmansky) and University of California, San Diego, La Jolla (Dr Desikan); Computer Science and Artificial Intelligence Laboratory, Massachusetts Institute of Technology (Drs Sabuncu and Fischl), Department of Psychology and Center for Brain Science, Harvard University (Drs Sepulcre, Yeo, and Buckner), and Howard Hughes Medical Institute (Drs Sepulcre and Buckner), Cambridge, Massachusetts; Center for Alzheimer Research and Treatment, Department of Neurology, Brigham and Women's Hospital, and Department of Neurology, Massachusetts General Hospital, Boston (Dr Sperling); and Department of Radiology, University of California, San Francisco, and Department of Veteran Affairs, San Francisco, California (Dr Weiner).

Correspondence: Mert R. Sabuncu, PhD, Department of Radiology, Athinoula A. Martinos Center for Biomedical Imaging, Massachusetts General Hospital, Bldg 149, 13th Street, Room 2301, Charlestown, MA 02129 (msabuncu@csail.mit.edu).

Author Contributions: Study concept and design: Sabuncu, Desikan, Liu, Reuter, and Weiner. Acquisition of data: Weiner and Sperling. Analysis and interpretation of data: Sabuncu, Desikan, Sepulcre, Yeo, Schmansky, Buckner, Sperling, and Fischl. Drafting of the manuscript: Sabuncu, Desikan, and Fischl. Critical revision of the manuscript for important intellectual content: Sabuncu, Desikan, Sepulcre, Yeo, Liu, Schmansky, Reuter, Weiner, Buckner, Sperling, and Fischl. Statistical analysis: Sabuncu, Yeo, Buckner, and Fischl. Obtained funding: Sabuncu, Weiner, and
Fischl. Administrative, technical, and material support: Sabuncu, Liu, Schmansky, Reuter, Weiner, and Fischl. Study supervision: Desikan, Buckner, Sperling, and Fischl.

Financial Disclosure: Dr Weiner has served on scientific advisory boards for CoMentis Inc, Neurochem International Limited, Elan and Wyeth Alzheimer's Immunotherapy Program (North America), Novartis International AG, Banner Alzheimer's Institute Alzheimer's Prevention Initiative, Eli Lilly and Company, Araclon Biotech, Institut Català de Neurociències Aplicades, Research Advisory Committee on Gulf War Veterans' Illnesses, Biogen Idec, Pfizer Global Research \& Development, Forest Laboratories Inc, Medivation Inc, Bayer Schering-Plough Corporation, Elan Pharmaceuticals Inc, Ipsen Group, Daiichi Sankyo Inc, AstraZeneca AB, TauRx Therapeutics Ltd, Bayer Healthcare, Exonhit Therapeutics SA, Servier Laboratories, and SYNARC Inc. He has received funding for travel from the Foundation for the National Institutes of Health (NIH); Avid Radiopharmaceuticals Inc; GE Healthcare Japan; the Research Association for Biotechnology; Pfizer Global Research \& Development; Forest Laboratories Inc; Alliance Médicale et Scientifique; the National Multiple Sclerosis Society; Bayer Healthcare; The Johns Hopkins University; the Alzheimer's Association; the Telemedicine \& Advanced Technology Research Center; the University of Pittsburgh; the Neuroscience Early Stage Research Training Program; the Rotman Research Institute; the Wien Center for Alzheimer's Disease and Memory Disorders; Mount Sinai Medical Center; the Society of Photographic Instrumentation Engineers; the Montreal Neurological Institute and Hospital of McGill University; the Michael J. Fox Foundation for Parkinson's Research; Elan and Wyeth Alzheimer's Immunotherapy Program (North America); Forest Laboratories Inc; the University of California, Davis; Tel Aviv University Medical School; Colloquium Paris; Ipsen Group; the Wenner-Gren Foundation; the US Social Security Administration; the Korean Neurological Association; the NIH; Washington University in St Louis; Banner Alzheimer's Institute; Clinical Trials on Alzheimer's Disease; the Veterans Affairs Central Office; the Beijing Institute of Geriatrics; Innogenetics NV; New York University; NeuroVigil Inc; Centre Hospitalier Régional Universitaire-Hôpital Roger Salengro; Siemens AG; AstraZeneca AB; Geneva University Hospitals; Eli Lilly and Company; Hôpital Pitié-Salpêtrière (Assistance Publique-Hôpitaux de Paris); Institut Català de Neurociències Aplicades; the University of New Mexico School of Medicine; and Clinical Trials on Alzheimer's Disease. He has served on the editorial board for Alzheimer's \& Dementia, has received honoraria from GE Healthcare Japan, Research Association for Biotechnology, The Johns Hopkins University, the Rotman Research Institute, the American Academy of Neurology, Ipsen Group, NeuroVigil Inc, and Institut Català de Neurociències Aplicades; has received commercial entities research support from Merck \& Co Inc and Avid Radiopharmaceuticals Inc; has received government entities research support from the NIH, the US Department of Defense, and the US Department of Veterans Affairs; and has stock options in SYNARC Inc and Elan Pharmaceuticals Inc. 
Funding/Support: Data collection and sharing for this project was funded by the ADNI and NIH grant U01 AG024904 (principal investigator [PI], Dr Weiner). The ADNI is funded by the National Institute on Aging, by the National Institute of Biomedical Imaging and Bioengineering, and through generous contributions from $\mathrm{Ab}$ bott Laboratories, AstraZeneca AB, Bayer Schering Pharma AG, Bristol-Myers Squibb, Eisai Global Clinical Development, Elan Corporation plc, Genentech Inc, GE Healthcare, GlaxoSmithKline plc, Innogenetics NV, Johnson \& Johnson Services Inc, Eli Lilly and Company, Medpace Inc, Merck \& Co Inc, Novartis International AG, Pfizer Inc, F. Hoffman-La Roche Ltd, Schering-Plough Corporation, SYNARC Inc, and Wyeth Pharmaceuticals, as well as nonprofit partners the Alzheimer's Association and the Alzheimer's Drug Discovery Foundation, with participation from the US Food and Drug Administration. Private sector contributions to the ADNI are facilitated by the Foundation for the NIH. The grantee organization is the Northern California Institute for Research and Education Inc, and the study is coordinated by the Alzheimer's Disease Cooperative Study at the University of California, San Diego. The ADNI data are disseminated by the Laboratory of Neuro Imaging at the University of California, Los Angeles. Further support for this research was provided in part by grant P4l-RR14075 from the National Center for Research Resources (Dr Fischl [PI, Bruce Rosen, MD, PhD]), grant R01EB006758 from the National Institute for Biomedical Imaging and Bioengineering (PI, Dr Fischl), grant AG022381 from the National Institute on Aging (Dr Fischl [PI, William S. Kremen, $\mathrm{PhD}$ ]), grant R01 NS052585-01 from the National Institute for Neurological Disorders and Stroke (PI, Dr Fischl), grant P41-RR14075 from the National Center for Research Resources (Dr Fischl [PI, Dr Rosen]), grants BIRN002 and U24 RR021382 from the National Center for Research Resources Biomedical Informatics Research Network Morphometric Project (Dr Fischl [PI, Dr Rosen]), grant RC1 AT005728-01 from the National Center for Alternative Medicine (Dr Fischl [PI, Dr Rosen]), grant R01EB006758 from the National Institute for Biomedical Imaging and Bioengineering (PI, Dr Fischl), and grant R01 NS052585-01 from the National Institute for Neurological Disorders and Stroke (PI, Dr Fischl); from Shared Instrumentation Grants 1S10RR023401, 1S10RR019, and 1S10RR023043; and The Autism \& Dyslexia Project funded by the Ellison Medical Foundation. Dr Sabuncu receives support from a KL2 Medical Research Investigator Training grant awarded via Harvard Catalyst, The Harvard Clinical and Translational Science Center (NIH grant 1KL2RR025757-01 and financial contributions from Harvard University and its affiliated academic health care centers).

Additional Contributions: Data used in the preparation of this article were obtained from the ADNI database (http: //adni.loni.ucla.edu/). As such, the investigators within the ADNI contributed to the design and implementation of the ADNI and/or provided data but did not participate in the analysis or writing of this report.
1. Braak H, Braak E. Neuropathological staging of Alzheimer-related changes. Acta Neuropathol. 1991;82(4):239-259.

2. Arnold SE, Hyman BT, Flory J, Damasio AR, Van Hoesen GW. The topographical and neuroanatomical distribution of neurofibrillary tangles and neuritic plaques in the cerebral cortex of patients with Alzheimer's disease. Cereb Cortex. 1991; 1(1):103-116.

3. Fox NC, Freeborough PA. Brain atrophy progression measured from registered serial MRI: validation and application to Alzheimer's disease. J Magn Reson Imaging. 1997;7(6):1069-1075.

4. Fox NC, Scahill RI, Crum WR, Rossor MN. Correlation between rates of brain atrophy and cognitive decline in AD. Neurology. 1999;52(8):1687-1689.

5. Jack CR Jr, Shiung MM, Gunter JL, et al. Comparison of different MRI brain atrophy rate measures with clinical disease progression in AD. Neurology. 2004; 62(4):591-600.

6. Jack CR Jr, Shiung MM, Weigand SD, et al. Brain atrophy rates predict subsequent clinical conversion in normal elderly and amnestic MCI. Neurology. 2005; 65(8):1227-1231.

7. Jack CR Jr, Weigand SD, Shiung MM, et al. Atrophy rates accelerate in amnestic mild cognitive impairment. Neurology. 2008;70(19, pt 2):1740-1752.

8. Holland D, Brewer JB, Hagler DJ, Fennema-Notestine C, Dale AM; Alzheimer's Disease Neuroimaging Initiative. Subregional neuroanatomical change as a biomarker for Alzheimer's disease [published correction appears in Proc Natl Acad Sci U S A. 2010;107(14):6551]. Proc Natl Acad Sci U S A. 2009;106(49):2095420959.

9. Whitwell J, Przybelski S, Weigand S, et al. 3D maps from multiple MRI illustrate changing atrophy patterns as subjects progress from mild cognitive impairment to Alzheimer's disease. Brain. 2007;130(pt 7):1777-1786.

10. Fox NC, Warrington EK, Freeborough PA, et al. Presymptomatic hippocampal atrophy in Alzheimer's disease: a longitudinal MRI study. Brain. 1996;119(pt 6): 2001-2007.

11. Scahill RI, Schott JM, Stevens JM, Rossor MN, Fox NC. Mapping the evolution of regional atrophy in Alzheimer's disease: unbiased analysis of fluid-registered serial MRI. Proc Natl Acad Sci U S A. 2002;99(7):4703-4707.

12. Schuff N, Woerner N, Boreta L, et al. MRI of hippocampal volume loss in early Alzheimer's disease in relation to ApoE genotype and biomarkers. Brain. 2009; 132(pt 4):1067-1077

13. Frisoni G, Fox N, Jack C, Scheltens P, Thompson PM. The clinical use of structural MRI in Alzheimer disease. Nat Rev Nephrol. 2010;6(2):67-77.

14. Ridha BH, Barnes J, Bartlett JW, et al. Tracking atrophy progression in familial Alzheimer's disease: a serial MRI study. Lancet Neurol. 2006;5(10):828-834.

15. Narr KL, Toga AW, Szeszko P, et al. Cortical thinning in cingulate and occipital cortices in first episode schizophrenia. Biol Psychiatry. 2005;58(1):32-40.

16. Shaw $P$, Kabani NJ, Lerch JP, et al. Neurodevelopmental trajectories of the human cerebral cortex. J Neurosci. 2008;28(14):3586-3594.

17. Jack CR Jr, Petersen RC, Xu YC, et al. Medial temporal atrophy on MRI in normal aging and very mild Alzheimer's disease. Neurology. 1997;49(3):786-794.

18. Chan D, Janssen JC, Whitwell JL, et al. Change in rates of cerebral atrophy over time in early-onset Alzheimer's disease: Iongitudinal MRI study. Lancet. 2003; 362(9390):1121-1122.

19. Rusinek H, Endo Y, De Santi S, et al. Atrophy rate in medial temporal lobe during progression of Alzheimer disease. Neurology. 2004;63(12):2354-2359.

20. Sluimer JD, van der Flier WM, Karas GB, et al. Accelerating regional atrophy rates in the progression from normal aging to Alzheimer's disease. Eur Radiol. 2009; 19(12):2826-2833

21. Amieva H, Jacqmin-Gadda $\mathrm{H}$, Orgogozo J-M, et al. The 9 year cognitive decline before dementia of the Alzheimer type: a prospective population-based study. Brain. 2005;128(pt 5):1093-1101.

22. Stern Y, Liu X, Albert M, et al. Application of a growth curve approach to modeling the progression of Alzheimer's disease. J Gerontol A Biol Sci Med Sci. 1996; 51(4):M179-M184

23. Jack CR Jr, Knopman DS, Jagust WJ, et al. Hypothetical model of dynamic biomarkers of the Alzheimer's pathological cascade. Lancet Neurol. 2010;9(1): 119-128.

24. Caroli A, Frisoni GB; Alzheimer's Disease Neuroimaging Initiative. The dynamics of Alzheimer's disease biomarkers in the Alzheimer's Disease Neuroimaging Initiative cohort. Neurobiol Aging. 2010;31(8):1263-1274.

25. Bass F. Comments on "A New Product Growth for Model Consumer Durables: The Bass Model." Manage Sci. 2004;50(12):1833-1840.

26. Sabuncu MR, Desikan RS, Sepulcre J, et al. The cumulative diffusion model. Harvard University Web site. ftp://surfer.nmr.mgh.harvard.edu/pub/articles /Sabuncu_ArchivesOfNeurology_2011_Supplemental.pdf. Accessed October 27, 2010. 
27. Shaw LM, Vanderstichele H, Knapik-Czajka M, et al; Alzheimer's Disease Neuroimaging Initiative. Cerebrospinal fluid biomarker signature in Alzheimer's Disease Neuroimaging Initiative subjects. Ann Neurol. 2009;65(4):403-413.

28. Fagan A, Roe C, Xiong C, Mintun M, Morris J, Holtzman D. Cerebrospinal fluid tau/ $\beta$-amyloid ${ }_{42}$ ratio as a prediction of cognitive decline in nondemented older adults. Arch Neurol. 2007;64(3):343-349.

29. Morris JC. The Clinical Dementia Rating (CDR): current version and scoring rules. Neurology. 1993;43(11):2412-2414.

30. Petersen RC. Mild cognitive impairment as a diagnostic entity. J Intern Med. 2004 256(3):183-194.

31. Fischl B, Sereno MI, Dale AM. Cortical surface-based analysis, II: inflation, flattening, and a surface-based coordinate system. Neuroimage. 1999;9(2):195207.

32. Fischl $B$, Dale AM. Measuring the thickness of the human cerebral cortex from magnetic resonance images. Proc Natl Acad Sci U S A. 2000;97(20):1105011055.

33. Fischl B, Salat DH, Busa E, et al. Whole brain segmentation: automated labeling of neuroanatomical structures in the human brain. Neuron. 2002;33(3):341355.

34. Buckner RL, Head D, Parker J, et al. A unified approach for morphometric and functional data analysis in young, old, and demented adults using automated atlasbased head size normalization: reliability and validation against manual measurement of total intracranial volume. Neuroimage. 2004;23(2):724-738.

35. Marcus DS, Wang TH, Parker J, Csernansky JG, Morris JC, Buckner RL. Open
Access Series of Imaging Studies (OASIS): cross-sectional MRI data in young, middle aged, nondemented, and demented older adults. J Cogn Neurosci. 2007; 19(9):1498-1507.

36. Fischl B, Sereno MI, Tootell RBH, Dale AM. High-resolution intersubject averaging and a coordinate system for the cortical surface. Hum Brain Mapp. 1999; 8(4):272-284.

37. Folstein MF, Robins LN, Helzer JE. The Mini-Mental State Examination. Arch Gen Psychiatry. 1983;40(7):812.

38. Draper NR, Smith H. Applied Regression Analysis. New York: John Wiley \& Sons; 1966.

39. Dickerson BC, Bakkour A, Salat DH, et al. The cortical signature of Alzheimer's disease: regionally specific cortical thinning relates to symptom severity in very mild to mild $A D$ dementia and is detectable in asymptomatic amyloid-positive individuals. Cereb Cortex. 2009;19(3):497-510.

40. Desikan RS, Sabuncu MR, Schmansky NJ, et al; Alzheimer's Disease Neuroimaging Initiative. Selective disruption of the cerebral neocortex in Alzheimer's disease. PLoS One. 2010;5(9):e12853. doi:10.1371/journal.pone.0012853.

41. Becker JA, Hedden T, Carmasin J, et al. Amyloid- $\beta$ associated cortical thinning in clinically normal elderly [published online November 17, 2010]. Ann Neurol. doi:10.1002/ana.22333/full.

42. Mormino EC, Kluth JT, Madison CM, et al; Alzheimer's Disease Neuroimaging Initiative. Episodic memory loss is related to hippocampal-mediated $\beta$-amyloid deposition in elderly subjects. Brain. 2009;132(pt 5):1310-1323.

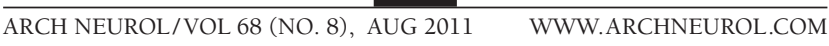

1048

Downloaded from www.archneurol.com at National University of Singapore, on September 6, 2011

@2011 American Medical Association. All rights reserved. 